\title{
The course of early disinhibited social engagement among post-institutionalized adopted children
}

\author{
Jamie M. Lawler, ${ }^{1}$ Kalsea J. Koss, ${ }^{2}$ Colleen M. Doyle, ${ }^{3}$ and Megan R. Gunnar ${ }^{3}$ \\ ${ }^{1}$ Department of Psychiatry, University of Michigan, Ann Arbor, MI; ${ }^{2}$ Department of Psychology and Human \\ Development, Vanderbilt University, Nashville, TN; ${ }^{3}$ Institute of Child Development, University of Minnesota, \\ Minneapolis, MN, USA
}

\begin{abstract}
Background: Approximately 20\% of post-institutionalized (PI) children exhibit disinhibited social engagement (DSE) or the propensity to approach and engage strangers. There is little longitudinal research examining changes in DSE after adoption, or methods of identifying children with persistent behaviors. Methods: DSE was assessed observationally four times during the first 2 years postadoption in PI children 16-36 months at adoption $(n=68)$ relative to same-age nonadopted children $(n=52)$. At age 5 , a validated interview determined which PI children met criteria for Disinhibited Social Engagement Disorder (DSED). Results: DSE trajectories initially increased and then stabilized. PIs had higher DSE levels initially and a steeper increase rate than NAs. When separated into physical and nonphysical DSE components, group differences arose in initial physical DSE and the rate of change of nonphysical DSE. DSE rate of increase predicted DSED diagnosis, as did longer institutional duration and poorer institutional care. Conclusions: The rate of increase in DSE postadoption, rather than the level observed at adoption, is predictive of disordered social engagement by age 5 years. Keywords: Adoption; attachment disorders; deprivation; developmental psychopathology; social behavior.
\end{abstract}

\section{Introduction}

Children who experience early adversity are at heightened risk for negative social, cognitive, and behavioral outcomes. This has been noted for post-institutionalized (PI) children who experience sometimes profound physical, social, and emotional deprivation prior to adoption (Smyke et al., 2007). Despite significant improvements in functioning following adoption or fostering, long-term outcomes in PI children range widely. Disinhibited social engagement (DSE; also known as indiscriminate friendliness, Chisholm, 1998), with significant clinical impacts on social and academic achievement, is observed in approximately one fifth of PI children (DSM-5; American Psychiatric Association, 2013; Rutter et al., 2007). DSE is the core feature of Disinhibited Social Engagement Disorder (DSED). There is little longitudinal research and no established method for measuring DSE, or do we know which children exhibiting DSE behaviors at adoption will continue on to reach criteria for disorder. The present study sought to fill this gap by testing whether individual differences in DSE behaviors measured by observational codes beginning several months postadoption predict DSED assessed using a validated interview at age 5 years. DSE behaviors in PI children were compared to those in agematched children born and reared in families comparable in socioeconomic class to families who adopt internationally. This study builds on previous reports of DSE behaviors on these children from

Conflict of interest statement: No conflicts declared. the first two assessments postadoption (Lawler, Hostinar, Mliner, \& Gunnar, 2014).

\section{Conceptualization and correlates}

The core features of DSED are a general lack of developmentally appropriate reticence around unfamiliar adults, a failure to check back with a caregiver in novel situations, and a tendency to wander off (APA, 2013). Stranger reticence is a normative developmental milestone that appears between 6 and 12 months of age (Brooker et al., 2013; Sroufe, 1977). While typically developing children exhibit variability in stranger reticence, children exhibiting DSED show little or no wariness of strangers and instead approach and engage unfamiliar adults readily, transgressing appropriate physical and verbal boundaries (Rutter et al., 2007).

DSED was separated from Reactive Attachment Disorder (RAD) in DSM-5 due to evidence that they represent distinct conditions (APA, 2013). Both RAD and DSED arise from neglect, and can be readily identified in institutionalized children (Zeanah, Smyke, \& Dumitrescu, 2002; Zeanah, Smyke, Koga, $\&$ Carlson, 2005) and maltreated children (Pears, Fisher, Bruce, Kim, \& Yoerger, 2010; Zeanah et al., 2004). However, significant differences between RAD and DSED correlates and response to intervention point to distinct pathologies (for review see Zeanah \& Gleason, 2015). RAD is tied closely to attachment and dissipates when children exit conditions of social deprivation (Smyke et al., 2012). RAD rarely is observed in clinical samples characterized by typical caregiving, including children assessed after adoption (O'Connor \& Rutter, 2000). In contrast, the 
connection between DSED and attachment has been widely debated. Current research suggests that DSED arises in the context of social neglect and persists after adoption or fostering, but is independent of attachment relationships with foster or adoptive parents (Chisholm, 1998; Gleason et al., 2011; Smyke, Zeanah, Fox, Nelson, \& Guthrie, 2010; Tizard, 1977). DSED symptoms are moderately stable into adolescence (Kreppner et al., 2010; Rutter et al., 2007). A deficit in self-regulation and executive control may contribute to the course of DSED (Bruce, Tarullo, \& Gunnar, 2009; Roy, Rutter, $\&$ Pickles, 2004). DSE may represent an enduring detrimental effect of nonoptimal neurobehavioral development as a result of early social deprivation (Bruce et al., 2009). Despite a sex difference in other disorders characterized by a lack of inhibitory control (e.g. ADHD; Gershon, 2002), sex has not been associated with DSE in PI samples (Gleason et al., 2011; Rutter et al., 2007; Zeanah et al., 2002).

Notably, the association between the length of institutionalization and DSE has been mixed. Some studies have found that duration of institutionalization, but not degree of physical deprivation or maltreatment, predicts severity of DSE (O'Connor \& Rutter, 2000). Some note an association between DSE and the quality of social-emotional caregiving (i.e. sensitivity by caregivers; Smyke, Dumitrescu, \& Zeanah, 2002); however, others have not (Zeanah et al., 2005). Approximately $17-32 \%$ of children exposed to severe social deprivation meet diagnostic criteria (Gleason et al., 2011), while the disorder rarely is observed in other clinical settings (APA, 2013). When disinhibited behavior is examined on a continuum, children adopted from institutions after 6 months of age exhibit significantly more DSE than family-reared or early-adopted children, as measured by parent-report (Chisholm, 1998; O'Connor, Bredenkamp, \& Rutter, 1999; O'Connor \& Rutter, 2000) and observational procedures (Bruce et al., 2009). Nevertheless, some children institutionalized for longer durations showed no evidence of DSE (O'Connor \& Rutter, 2000; Zeanah et al., 2005). A recent examination of children randomized to foster-care following institutionalization, found only attachment disorganization prior to randomization predicted DSE at 54 months (Gleason et al., 2014).

\section{Measurement and course}

Previous research has used parent interview and behavioral observations to measure DSED. Parent interviews have shown acceptable reliability and internal consistency, but are subject to reporter bias (Gleason et al., 2011; Rutter et al., 2007; Zeanah et al., 2004, 2005). Behavioral observations are more objective and can be used repeatedly to assess changes in DSE behaviors but are only moderately correlated with parent-report (Gleason et al., 2011). Previously, we assessed physical contact (touching an unfamiliar adult) and nonphysical contact (distal engagement of the adult) measures of DSE soon after adoption. We found physical contact DSE behaviors differentiated PI from nonadopted 18- to 37-monthold children better than nonphysical DSE (Lawler et al., 2014). Physical DSE decreased over the first-year postadoption, while nonphysical DSE increased.

\section{Clinical importance}

DSED is associated with deficits in socioemotional competence, functional impairments, inattention and hyperactivity, externalizing behavior, and peer relational abnormalities (Gleason et al., 2011; Hodges \& Tizard, 1989; Lyons-Ruth, Bureau, Riley, \& Atlas-Corbett, 2009; Roy et al., 2004). Children who meet criteria for DSED utilize more special education and mental health services (Rutter et al., 2007) and are at greater risk of developing emotional and conduct problems (Rutter et al., 2010). DSE behaviors are potentially dangerous for the children (e.g. leaving with strangers) and deleterious to the caregiver-child relationship if caregivers feel they signify a lack of attachment (Albus \& Dozier, 1999).

\section{Current study}

While some children show continuously elevated DSE, others demonstrate remittance in DSE (Rutter et al., 2007, 2010). Currently, we have little predictive understanding of which children will continue or remit. Rutter et al. (2007) examined persistence of DSE in PI children from 6 to 11 years. Persistent DSE behaviors were associated with higher initial DSE, smaller head circumference, and more inattention/overactivity symptoms. None of the postadoption environmental factors tested differentiated persistence versus remission, including adoptive parents' education, cognitive abilities, mental health, or quality or stability of the marital/romantic relationship. Duration of deprivation continued to predict DSE; however, behavior soon after adoption was not examined. Identification of DSED-predictive behaviors appearing early postadoption would allow for early and targeted intervention.

The present study examined trajectories of DSE behaviors across the first several years postadoption, extending our previous report on the first 8 months (Lawler et al., 2014). PI children were compared to children born and reared in families of similar education and income to adoptive families. The period of development examined, from toddlerhood through the preschool period, is one of the changes in children's sociability, verbal abilities, and self-regulation. In American culture, shy children are encouraged to be more outgoing when meeting new adults in their parent's presence, while socially outgoing children are trained to be more constrained to conform to cultural expectations. Thus, the course 
of DSE behaviors in PI children needs to be judged against the normative changes in children who have not lacked early attachment figures. DSE behaviors in the months postadoption were used to predict DSED at age 5 only in the PI children, given evidence that DSED would be nonexistent in the low-risk comparison children (Garvin, Tarullo, Van Ryzin, \& Gunnar, 2012). Individual differences in preadoption adversity were also examined as predictors of DSE trajectories and DSED.

\section{Method Participants}

Sixty-eight PI $(60 \%$ female; adopted between 16 and 36 months) and 52 nonadopted (NA; 50\% female) children were included (see Table 1). PI children were recruited from Midwestern adoption clinics and agencies. Age-matched NA children were recruited from a list of families interested in research participation. Participants were part of a larger project examining recovery in PI children. All families provided informed consent, as approved by the institutional review board. Primary caregivers participated in all sessions $(>90 \%$ mothers). Additional recruitment and exclusion criteria are available in Appendix S1.

\section{Procedure}

Four in-person, parent-child laboratory assessments (T1 to T4) and two telephone interviews (one preadoptive care interview; one clinical interview) were conducted. T1 occurred soon after adoption $(M=1.7$ months, $S D=0.8$; range $=0.33-3.99)$, with subsequent sessions at 8 -month intervals (see Table 1). The DSE observation assessment was identical across T1-T4. Change from T1 to T2 was reported in Lawler et al., 2014. The preadoptive care interview occurred within the first-year postadoption, while the DSED interview occurred when PI children were age 5 .

An observational method for evaluating DSE behaviors was used during each laboratory session. Briefly, a scripted female stranger interaction derived from Tizard and Rees (1975) was videotaped and later coded by observers blind to adoption history. The caregiver completed paperwork and was discouraged from interacting with the child or influencing his/her behavior toward the stranger. The stranger entered the playroom and made increasing social overtures (i.e. greeting the child, offering toys) at scripted intervals, culminating in interactive play (see Appendix S2 for details).

\section{Measures}

Observed DSE. The 10-minute stranger interaction was videotaped and later coded (Lawler et al., 2014) using the ProCoder program (Tapp, 2003). Children's verbal and nonverbal initiations and their proximity and physical contact to the stranger were scored. Child initiations were tallied in order to capture the child's attempts to engage the stranger. Proximity within two feet of the stranger was coded for frequency of approach and duration. Physical contact included childinitiated direct contact with the stranger assessed by frequency, duration, and overall degree throughout the task. Latency to first initiation, approach, and touch were noted. Coders also rated the child's initial reaction to the stranger's greeting, toy offering, and play engagement, as well as overall

Table 1 Participant characteristics

\begin{tabular}{|c|c|c|c|c|c|c|}
\hline & \multicolumn{2}{|c|}{ PI $(n=68)$} & \multirow[b]{2}{*}{ Range } & \multirow[b]{2}{*}{ Time Since Adoption, $M$} & \multicolumn{2}{|c|}{ NA $(n=52)$} \\
\hline & $M$ & $S D$ & & & $M$ & $S D$ \\
\hline Length of institutionalization & 17.86 & 7.49 & $4.00-34.00$ & - & - & - \\
\hline \multicolumn{7}{|l|}{ Age } \\
\hline At adoption & 24.61 & 4.98 & $16.70,36.13$ & - & - & - \\
\hline $\mathrm{T} 1$ & 26.31 & 4.99 & $18.97,36.66$ & 1.70 & 27.65 & 5.71 \\
\hline T2 & 32.72 & 5.10 & $24.69,44.25$ & 8.27 & 34.68 & 5.80 \\
\hline T3 & 40.47 & 4.82 & $32.71,51.58$ & 16.23 & 42.73 & 5.82 \\
\hline T4 & 48.35 & 4.80 & $40.54,59.97$ & 24.12 & 50.76 & 5.58 \\
\hline T5 Interview & 61.32 & 1.38 & $59.87,65.39$ & 37.29 & - & - \\
\hline \multicolumn{5}{|c|}{ Median } & \multicolumn{2}{|c|}{ Median } \\
\hline \multirow[t]{2}{*}{ Family income } & \multicolumn{4}{|c|}{$\$ 100,000-125,000$} & \multicolumn{2}{|c|}{$\$ 75,000-100,000$} \\
\hline & \multicolumn{3}{|c|}{ Percent } & & \multicolumn{2}{|c|}{ Percent } \\
\hline \multicolumn{7}{|l|}{ Region of origin } \\
\hline Southeast Asia & \multicolumn{2}{|c|}{34} & & & & 0 \\
\hline Africa & \multicolumn{2}{|c|}{32} & & & & 0 \\
\hline Russia/Eastern Europe/India & \multicolumn{2}{|c|}{25} & & & & 0 \\
\hline Latin America & \multirow{2}{*}{\multicolumn{2}{|c|}{$\begin{array}{l}9 \\
0\end{array}$}} & & & \multirow{2}{*}{\multicolumn{2}{|c|}{$\begin{array}{r}0 \\
100\end{array}$}} \\
\hline United States & & & & & & \\
\hline \multicolumn{7}{|l|}{ Race } \\
\hline Asian & \multicolumn{2}{|c|}{41} & & & & 4 \\
\hline African/Black & \multicolumn{2}{|c|}{34} & & & & 0 \\
\hline Caucasian & \multicolumn{2}{|c|}{15} & & & & 89 \\
\hline Latin American Indian & \multicolumn{2}{|c|}{4} & & & & 0 \\
\hline Two or more races & \multirow{2}{*}{\multicolumn{2}{|c|}{$\begin{array}{l}3 \\
3\end{array}$}} & & & & 8 \\
\hline Other/unknown & & & & & & 0 \\
\hline
\end{tabular}

All ages and durations reported in months. 
verbal and physical intimacy. Interrater reliability $(20 \%$ of observations) averaged kappas of .71-.98 across 12 coders. Separately, laboratory experimenters rated each child for verbal and physical intimacy over the entire length of the 2$\mathrm{hr}$ session using the same coding scale (see Appendix S3 for details).

DSED Interview. An interview adapted from the Disturbances of Attachment Interview (Smyke et al., 2002) and a semistructured interview on attachment problems in PI children (O'Connor et al., 1999) was conducted involving 23 questions assessing RAD, DSED, and Separation Anxiety Disorder symptoms. Interviewers probed for specific example behaviors and asked follow-up questions to sufficiently characterize the child's behavior in a variety of situations. Interviewers used anchored criteria to make ratings. Each item was rated as ' 0 ' (not or rarely present), ' 1 ' (somewhat or sometimes present), or '2' (definitely or often present).

Separation anxiety was not a focus of the current investigation and was excluded from analysis. As expected, RAD symptoms were extremely rare in our sample (see Table 2) and the low base rate likely contributed to the scale's poor internal consistency $(\alpha=.36)$. Thus, RAD was not examined further.

Four ratings of DSED symptoms included: absence of reticence/willingness to go off with an unfamiliar adult [1], failure to check back with caregiver/tendency to wander off [2], and overly familiar verbal [3] and physical [4] behavior. The DSED scale had acceptable internal consistency $(\alpha=.76)$. Interrater reliability for the DSED scale was calculated on $24 \%$ of interviews (ICC range .72-.92). The mean of the four ratings was calculated resulting in scores ranging from 0 (no symptoms) to 2 (endorsed high levels of all symptoms). In line with DSM-5 diagnostic criteria for DSED (e.g. presence of $>2$ of 4 symptoms; APA, 2013), scores of 1 or above (indicating the presence of at least two symptoms) were used to create a dichotomous DSED diagnostic variable.

Early adversity. Preadoptive social care quality was rated by the interviewer on a 5-point scale (high = better) during phone interview during which parents described various aspects of caregiver-child interactions they observed in the institution (e.g. affection, interaction). Reliability was calculated on 10 interview scenarios between two coders (kappas > .80; see details in Appendix S4). To be independent of age-at-adoption, percentage of time in institutional care was calculated ( $r=.84, p<.001$ with total duration). Height-forage at first postadoption medical appointment $(z$-scored based on world health organization norms, Onis et al., 2007, see Table S1 for additional data) indexed severity of deprivationassociated growth-stunting.

\section{Data analytic plan}

Following descriptive statistics, latent change score (LCS) models (McArdle \& Hamagami, 2001) were fit examining group differences in early DSE trajectories. Four unconditional models were fit examining no change (stability in behaviors), constant change (linear growth), proportional change (change proportional to previous score), and dual change (both linear and proportional parameters) models. Nested models were compared using a chi-squared difference test. Group was included as a predictor in the selected model. Sex and T1 age were initially included as predictors of the latent intercept and slope factors; only significant covariates were retained. Separate models examined trajectories of physical and nonphysical DSE. Next, regression analyses tested if early DSE predicted age 5 DSED in PI children, including both categorical and dimensional approaches. Lastly, regression analyses and $t$ -

Table 2 Descriptive statistics

\begin{tabular}{lccc}
\hline & PI & NA & \\
& $M(S D)$ & $M(S D)$ & $F$ \\
\hline Early DSE & & & $F(1,114)=8.02^{* *}$ \\
T1 DSE Composite & $15.84(10.35)$ & $10.94(7.33)$ & $F(1,112)=3.12$ \\
T2 DSE Composite & $16.15(9.39)$ & $13.37(6.74)$ & $F(1,106)=7.56^{* *}$ \\
T3 DSE Composite & $17.54(9.77)$ & $13.08(6.20)$ & $F(1,104)=8.62^{* *}$ \\
T4 DSE Composite & $16.92(9.61)$ & $12.31(5.39)$ & $F(1,114)=17.83^{* * *}$ \\
T1 Physical DSE & $2.85(3.22)$ & $0.73(1.63)$ & $F(1,112)=4.36^{*}$ \\
T2 Physical DSE & $2.06(2.74)$ & $1.06(2.26)$ & $F(1,106)=10.48^{* *}$ \\
T3 Physical DSE & $2.28(2.82)$ & $0.79(1.66)$ & $F(1,104)=13.17^{* * *}$ \\
T4 Physical DSE & $1.71(2.48)$ & $0.34(0.84)$ & $F(1,114)=0.043$ \\
T1 Nonphysical DSE & $10.13(5.47)$ & $9.47(5.32)$ & $F(1,112)=0.64$ \\
T2 Nonphysical DSE & $12.02(5.48)$ & $11.25(4.64)$ & $F(1,106)=2.18$ \\
T3 Nonphysical DSE & $12.98(5.69)$ & $11.50(4.39)$ & $F(1,104)=2.89$ \\
T4 Nonphysical DSE & $13.49(6.17)$ & $11.63(4.82)$ & \\
\hline
\end{tabular}

\begin{tabular}{|c|c|c|c|}
\hline & \multicolumn{2}{|c|}{ PI } & \\
\hline & $M(S D)$ & Range & \\
\hline \multicolumn{4}{|l|}{ Early Adversity } \\
\hline Percent Preadoptive Life in Institution & $0.76(0.29)$ & $0.14-1.00$ & \\
\hline Social Care Quality & $3.14(1.40)$ & $1-5$ & \\
\hline \multirow[t]{2}{*}{ Growth-Stunting } & $-1.19(1.12)$ & $-4.21-0.76$ & \\
\hline & & & Percent above clinical cutoff \\
\hline \multicolumn{4}{|l|}{ Age 5 Assessment } \\
\hline DSED Symptoms & $0.45(0.52)$ & $0.00-2.00$ & $20.75 \%$ \\
\hline RAD Symptoms & $0.10(0.13)$ & $0.00-0.57$ & $1.89 \%$ \\
\hline
\end{tabular}

${ }^{*} p<.05 ;{ }^{* *} p<.01 ;{ }^{* * *} p<.001$. 
tests examined individual differences in early adversity as predictors of early DSE and DSED in PI children. (See Table S2 for examination of racial/ethnic differences in DSE/DSED).

\section{Results}

Descriptive statistics and ANOVA results depicting group differences in DSE at T1-T4 are displayed in Table 2. There were no significant sex differences (see Table S3). See Table S4 for correlations among early adversity variables.

\section{Growth trajectories of early DSE}

Unconditional LCS models were compared. The dual change model $\left(\chi^{2}(7)=13.73\right)$ fit the data significantly better than the no change $\left(\chi^{2}(11)=24.59\right.$; $\chi^{2}$ diff $\left.=10.86, \Delta d f=4, p<.05\right)$, constant change $\left(\chi^{2}(8)=20.39 ; \chi^{2}\right.$ diff $\left.=6.66, \Delta d f=1, p<.01\right)$, and proportional change $\left(\chi^{2}(10)=22.98 ; \chi^{2}\right.$ diff $=9.25$, $\Delta d f=3, p<.05)$ models for the composite DSE scale.

Group $(0=\mathrm{NA}, 1=\mathrm{PI})$ was a significant predictor of the intercept and slope in the dual change model and provided adequate fit $\left(\chi^{2}(9)=14.26, n s\right.$; $\mathrm{TLI}=.91 ;$ RMSEA $=.07)$. PIs had higher initial DSE and steeper rates of change (see Table 3 for full model results; Figure 1A group-specific trajectories).

\section{Physical and nonphysical early DSE}

Physical DSE. The unconditional proportional change $\left(\chi^{2}(10)=16.75\right)$ and the constant change $\left(\chi^{2}(8)=13.61\right)$ models fit the data significantly better than a no change model $\left(\chi^{2}(11)=25.56\right.$; $\chi^{2}$ diff $=8.81, \Delta d f=1, p<.01 ; \chi^{2}$ diff $=11.95, \Delta d f=$ $3, p<.01$, respectively). The proportional and constant change models are nonnested and the chisquare difference test cannot be used to make direct comparisons; the constant change model with predictors resulted in an inadmissible solution. Thus, the proportional change model was selected. While this model fit the data significantly better than a no change model, the conditional model provided inadequate model fit $\left(\chi^{2}(16)=22.20, \quad n s ; \quad\right.$ TLI $=.87$; RMSEA $=.06)$ and results should be interpreted

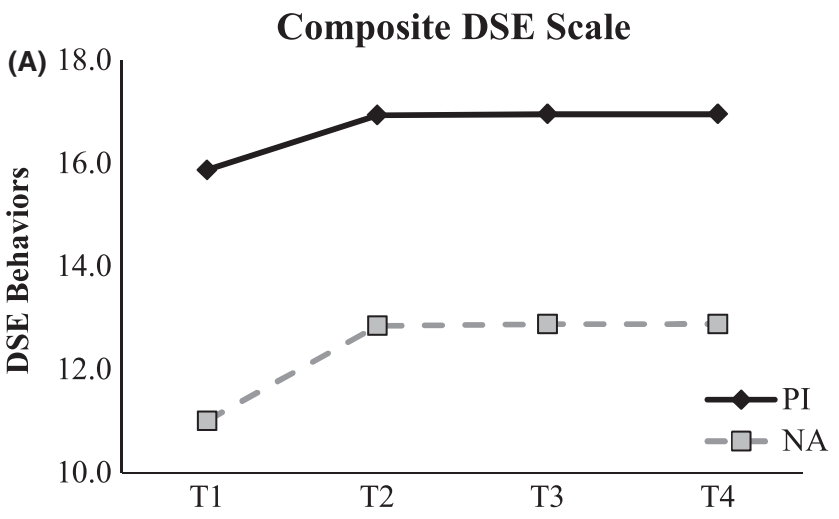

Physical DSE Scale

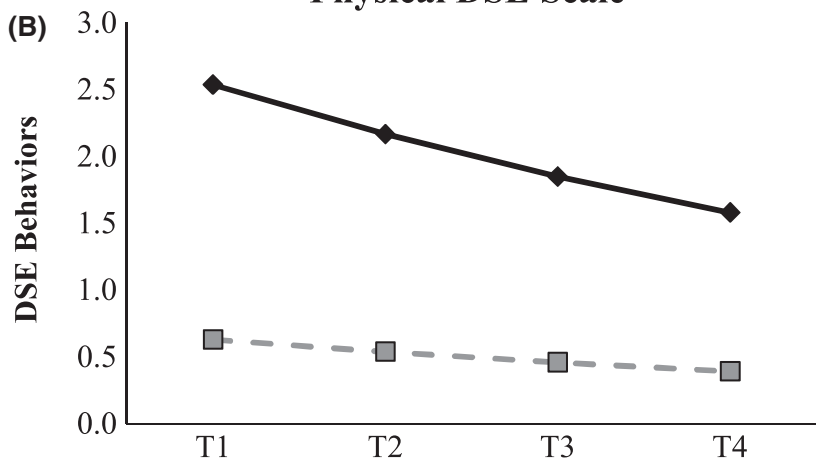

Non-Physical DSE Scale

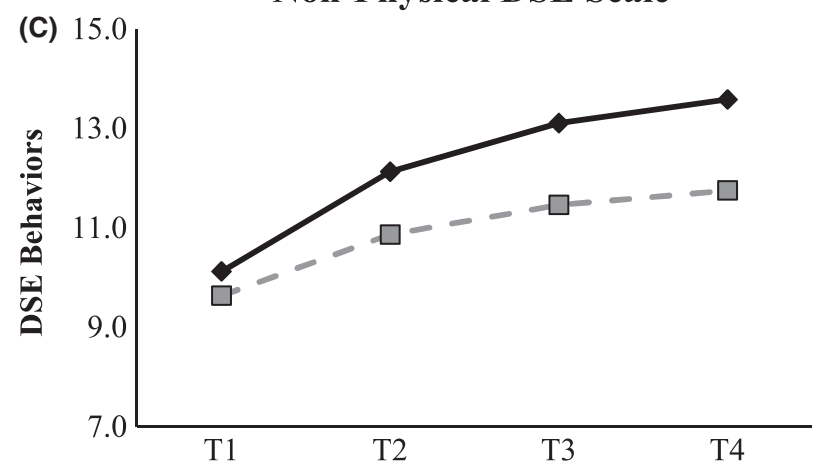

Figure $1 \mathrm{PI}$ and NA trajectories of early DSE behaviors

Table 3 Parameter estimates for LCS models of early DSE

\begin{tabular}{|c|c|c|c|c|c|c|}
\hline & \multicolumn{2}{|c|}{ DSE Composite } & \multicolumn{2}{|c|}{ Physical DSE } & \multicolumn{2}{|c|}{ Nonphysical DSE } \\
\hline & $B(S E)$ & $R^{2}$ & $B(S E)$ & $R^{2}$ & $B(S E)$ & $R^{2}$ \\
\hline \multicolumn{7}{|l|}{ Intercept } \\
\hline Mean & $11.00 * * *$ & .13 & $0.63^{*}$ & .86 & $9.64^{* * *}$ & $<.01$ \\
\hline Variance & $38.60 * * *$ & & 0.15 & & $13.95^{* * *}$ & \\
\hline \multicolumn{7}{|l|}{ Linear Slope } \\
\hline Mean & $12.66^{* * *}$ & .15 & - & & $136.18^{* * *}$ & .05 \\
\hline Variance & $21.92^{*}$ & & - & & $5.40^{*}$ & \\
\hline Proportional Change Parameter & $-0.98(0.22)^{* * *}$ & & $-0.15(.05)^{* *}$ & & $-0.51(0.16)^{* * *}$ & \\
\hline \multicolumn{7}{|l|}{ Predictors } \\
\hline Group $\rightarrow$ Intercept & $4.86(1.74)^{* *}$ & & $1.91(.31)^{* * *}$ & & $0.48(0.96)$ & \\
\hline Group $\rightarrow$ Linear Slope & $4.00(1.42)^{* *}$ & & - & & $1.03(0.57)^{\dagger}$ & \\
\hline Child Sex $\rightarrow$ Intercept & - & & $0.57(.29)^{*}$ & & - & \\
\hline
\end{tabular}

The physical DSE proportional change model does not include a linear growth parameter. Unstandardized estimates reported. ${ }^{\dagger} p<.07 ;{ }^{*} p<.05 ;{ }^{* *} p<.01 ;{ }^{* * *} p<.001$. 
with caution. Nonetheless, group and sex were significant predictors of initial physical DSE with PIs and boys having higher rates of T1 physical DSE (see Table 3 and Figure 1B; for sex-specific trajectories see Figure S1).

Nonphysical DSE. The dual change model $\left(\chi^{2}(7)=7.45\right)$ fit the data significantly better than the no change $\left(\chi^{2}(11)=51.74 ; \chi^{2}\right.$ diff $=44.29$, $\Delta d f=4, p<.001)$, constant change $\left(\chi^{2}(8)=13.89\right.$; $\chi^{2}$ diff $\left.=6.45, \Delta d f=1, \quad p<.01\right)$, and proportional change $\quad\left(\chi^{2}(10)=23.89 ; \quad \chi^{2}\right.$ diff $=16.44, \quad \Delta d f=3$, $p<.001)$ models. Group was included as a predictor in the dual change model and provided good fit $\left(\chi^{2}(9)=7.78, n s ;\right.$ TLI $=1.00 ;$ RMSEA $\left.=.00\right)$. There was a trend for group predicting differences in the linear slope $(p=.069)$ with nonphysical DSE behaviors becoming more divergent over the 2-year period (see Table 3 and Figure 1C).

\section{Age 5 DSED}

The intercept and linear slope parameters from the DSE composite model were used as predictors of age 5 DSED in PI children. Linear and logistic regressions examined continuous symptoms and a categorical variable $(0=$ doesn't meet criteria, $1=$ meets criteria).

DSED dimensional differences. T1 DSE behaviors (intercept) were not associated with age 5 DSED symptoms $(B=-0.01, \quad S E=0.02, n s)$. However, steeper change in early DSE was associated with heightened DSED symptoms $(B=0.04, S E=0.02$, $p<.05)$. Predictors accounted for a modest amount of variance in $\operatorname{DSED}\left(R^{2}=.10\right)$.

$D S E D$ categorical distinctions. Steeper change in early DSE was associated with a greater likelihood of meeting criteria for a DSED diagnosis $(\mathrm{B}=.24$, $S E=0.11, p<.05, \quad \mathrm{OR}=1.27) ; \mathrm{T} 1 \mathrm{DSE}$ did not predict DSED diagnosis $(\mathrm{B}=-.10, S E=0.10, n s$, $\mathrm{OR}=0.90 ;$ DSED $\left.R^{2}=.16\right)$.

Early DSE trajectories were examined by age 5 diagnostic status. DSED diagnosis was a significant predictor of the slope $(B=7.74, S E=2.34, p<.05)$ in a dual change model $\left(\chi^{2}(9)=8.06, n s\right.$; TLI $=1.00$; RMSEA $=.00)$. PI children meeting criteria increased in DSE following T1, whereas those without DSED evidenced lower, stable DSE (Figure 2).

\section{Early adversity and DSE and DSED}

Multiple regression analyses examined early adversity (social care quality, growth-stunting, percentage of life in an institution) as predictors of early DSE behaviors (intercept and slope) and DSED symptoms in PI children. Early adversity variables were not associated with initial or change in early DSE behaviors. Better social care was associated with

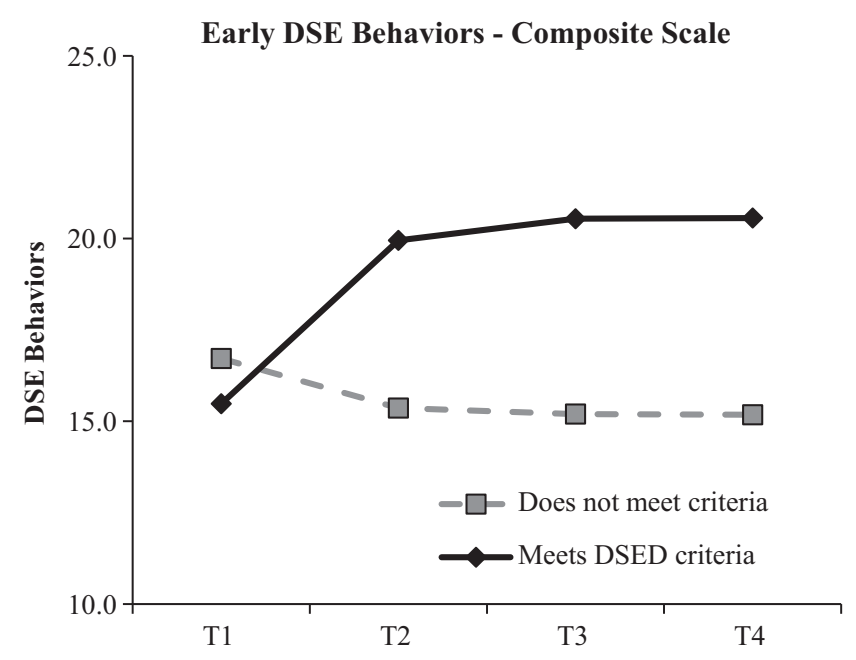

Figure 2 Trajectories of early DSE among PI children by age 5 DSED diagnostic criteria

fewer age 5 DSED symptoms $(\mathrm{B}=-.12, S E=0.05$, $p<.05)$; there were no associations between DSED symptoms and growth-stunting $(\mathrm{B}=.00, S E=0.06$, $n s)$ or percentage of life in an institution $(\mathrm{B}=.33$, $S E=0.23, n s)$. Early adversity variables accounted for a modest amount of variance in DSED symptoms $\left(R^{2}=.17\right)$, but not early DSE (intercept $R^{2}=.03$, slope $R^{2}=.05$ ).

$T$-tests examined differences in early adversity and DSED diagnosis. There were significant differences in social care $(t(38)=2.18, p<.05)$ and the percentage of life in an institution $(t(25.91)=-2.53, p<.05$, unequal variances assumed Levene's test $F(1$, $51)=7.94, p<.01)$, but not growth-stunting $(t$ $(51)=-.12, n s)$. Children meeting criteria for DSED spent a greater portion of their preadoptive life in an institution $(M=0.90, S D=0.18)$ and received poorer social care in the institution $(M=2.50, S D=1.41)$ compared to those not meeting criteria $(M=0.73$, $S D=0.29 ; M=3.17, S D=1.25$, respectively).

\section{Discussion}

We examined changes in observed social engagement for young children from two groups: children reared in their birth families and children adopted internationally from orphanages between 16 and 36 months. For PI children, we examined whether DSE behaviors soon after adoption and changes over the course of their transition to the family would prospectively identify children meeting criteria for DSED at age 5 .

\section{Change over time}

Both groups showed changes in these behaviors over the toddler and preschool years; specifically, both groups exhibited increasing nonphysical sociability and decreasing physical contact with strangers. However, the initial level and rate of change differed 
between groups. Physical contact readily differentiated PIs and NAs at T1. By the first assessment (1837 months), NA children already avoided initiating physical contact with unfamiliar, pleasant strangers. As a group, PI children's physical contact began to approach the NA children's level by the last assessment (40-60 months). Conversely, engaging with the stranger in nonphysical ways (e.g. asking questions, pointing, approaching but not touching) increased over the study in both groups. This likely reflects children's developmental gains in language and social engagement, as well as greater familiarity with the laboratory setting (but not the particular stranger). While sex was a significant predictor of the LCS model intercept, this was driven by sex differences in the NA children (see Table S3) and likely indicates temperamental contributions to nonphysical behaviors in typically developing children (see Lawler et al., 2014).

While both groups increased, PI children showed a more marked pattern of increase in nonphysical DSE behaviors. Group differences became more apparent by T4 (2 years postadoption; $M$ age $=4$ years). This escalation likely reflects increases in frequency of initiations and violation of verbal boundaries, such as sharing personal information or asking intrusive questions. Previously, we found these nonphysical DSE behaviors may not be problematic early in development (Lawler et al., 2014). However, these behaviors may become less socially acceptable as children age, and may serve to distinguish children with DSED after physical indices decline. While DSE behaviors increased, the difference between the groups remained relatively stable, indicating persistence, but not necessarily increase, in disordered behavior. This echoes a recent study showing no significant difference in DSE between children who were randomized to foster-care and those who remained institutionalized (Gleason et al., 2014). Cutting across our composite, physical, and nonphysical results, findings suggest that longitudinal assessment of DSE should be sensitive to capturing both types of behaviors.

\section{Predicting DSED}

Measures of T1 DSE ( $M=1.6$ months postadoption; 18-36 months old) were not predictive of age 5 DSED symptoms. However, observing changes over time, particularly from T1 to T2 $(M=8.2$ months postadoption; 26-44 months old), was informative. Children with a more marked increase in overall DSE were more likely to meet DSED diagnostic criteria at age 5 . As children had been in their new caregiving environment for 1-2 months on average at T1, DSE observed soon after adoption may reflect levels similar to those in institutions, or may be in flux due to the transition in caregivers upon adoption. This could be examined further by implementing more frequent assessments across the early postadoptive months to identify when the child's behavior stabilizes and becomes predictive of later outcomes following the major adjustments associated with cross-cultural adoption. Moreover, initial rank order relations may not relate to later disorder due to recovery in some of the children, independent from initial problems. By T2, children had been in their adopted homes for approximately 8 months and had established selective attachment relationships with primary caregivers (Carlson, Hostinar, Mliner, \& Gunnar, 2014). It is possible that aspects of the postadoption environment, including quality of parenting following adoption affect the rate of change of DSE.

\section{Preadoptive adversity}

Individual differences in the effect of early adversity on DSED were not apparent until later assessments ( $M=8.2$ months postadoption; 26-44 months old), suggesting that DSED differences may become more pronounced and unfold with development. This builds on our previous study which did not find any associations with preadoptive factors soon after adoption (Lawler et al., 2014). This pattern may be due to shifts in the expectation of developmentally and socially appropriate social behaviors. Alternatively, while the observation of DSE measured some behaviors associated with DSED, it did not capture a full range of situations pertinent to the disorder. For example, during the 10-minute interaction, there were no opportunities for the child to 'wander off'. Thus, a combination of observation, parent-report, and preadoptive risk together may be the most predictive of future disorder. Furthermore, the quality of social care, but not growth-stunting (an index of global deprivation), was associated with DSED, highlighting the importance of caregivers in preventing DSED. Notably, growth-stunting was not associated with other adversity measures, supporting the notion that this indexes a distinct form of deprivation. While duration of institutionalization was not linearly associated with DSED symptoms, children who met DSED criteria spent a significantly greater portion of their lives in an institution. Previous research on this association has been mixed and may be due to differences in measurement, analyses, or samples.

\section{Limitations and future directions for research, practice, and policy}

The current study had several limitations. First, as with most research in internationally adopted populations, we were unable to measure the preadoptive environment directly, control for variables such as size, staffing or resources of the institutions, or ascertain how earlier factors such as prenatal care or reasons for institutionalization might affect DSE (Oliveira et al., 2012). Similarly, we cannot be sure 
whether behaviors over this time are attributable to deprivation or to the significant transition of a crosscultural adoption. Second, we did not include measures of co-occurring symptomatology or functional impairment in the current analyses. DSE often overlaps with other problems that have been called 'deprivation-specific' patterns including attention problems (Rutter et al., 2010). In addition to DSE behaviors, attention and executive function difficulties are some of the most lasting effects of institutionalization, which may be the result of a sensitive window for development of these functions (Julian, 2013). Future research should examine if children with both DSE and attention regulation problems might benefit from earlier intervention. Furthermore, if attention and self-regulation are involved, interventions that target those deficits may in turn decrease DSE. A randomized trial of self-regulationfocused intervention is needed and would inform practice decisions for clinicians treating DSED. Third, we were unable to adequately examine race/ ethnicity due to the demographics of the NA children, as well as differences in preadoptive care that correspond to region/country of origin. Additional research is necessary to examine the role of race/ ethnicity in DSE. Fourth, because the DSED interview was conducted over the phone, we may have missed opportunities to probe for additional information indicated by nonverbal cues. Fifth, parentreported DSED symptoms may reflect bias, as parents are not blind to their child's developmental history. Continuing efforts to develop a multivariate assessment battery for DSED, which includes observational measures that can be blind-coded, can help minimize this potential source of bias.

This analysis did not examine the potential impact of the postadoption environment on DSE trajectories. While inadequate social care is implicated in the etiology of DSE, the high-quality parenting found in internationally adopting families does not ameliorate symptoms in all PI children. Research examining the postadoption environment has not found an effect on DSE between ages 6 and 11 (Rutter et al., 2007). However, in future analyses, we will consider specific aspects of parenting quality (sensitivity, responsiveness, limit-setting, providing structure) during the transition into the family that might influence trajectories of DSE behaviors.

Beyond reducing the number of children cared for in institutions, efforts should prioritize increasing social facets of care for institutionalized children. For children removed from depriving circumstances, intervention efforts should target those children who show steep increases in DSE behaviors in the year following placement in a supportive family.

\section{Supporting information}

Additional Supporting Information may be found in the online version of this article:

Appendix S1. Recruitment and exclusion criteria.

Appendix S2. DSE observation procedure.

Appendix S3. DSE coding scheme and data processing. Appendix S4. Preadoptive social care quality.

Table s1. Height-for-age data among PI and NA children.

Table S2. Racial differences in DSE behaviors and DSED symptoms among PI children.

Table S3. Sex-specific descriptive statistics among PI and NA children.

Table S4. Correlations among preadoptive adversity.

Figure S1. Trajectories of Early Physical DSE Behaviors among PI and NA boys and girls.

\section{Acknowledgements}

The authors thank the families for their participation and the International Adoption Project. Grant support was provided by R01 MH080905 and P50 MH078105 (to M.R.G.), by the Center for Neurobehavioral Development (University of Minnesota), and by NIMH training grant T32 MH018921 (to K.J.K.). The content is solely the responsibility of the authors and does not represent the views of the National Institutes of Health. The authors have declared that they have no competing or potential conflicts of interest in relation to this article.

\section{Correspondence}

Jamie M. Lawler, University of Michigan, 4250 Plymouth Rd. Ann Arbor, MI 48109, USA; Email: lawle084@umn.edu

\section{Key points}

- There is little longitudinal research examining changes in disinhibited social engagement (DSE) or methods of identifying children with persistent behaviors.

- DSE trajectories were characterized by initially increasing behaviors that became more stable.

- Differences were found between physical and nonphysical behaviors.

- The rate of increase in disinhibited behaviors postadoption, rather than the level observed at adoption, is predictive of age 5 disordered social engagement. 


\section{References}

Albus, K.E., \& Dozier, M. (1999). Indiscriminate friendliness and terror of strangers in infancy: Contributions from the study of infants in foster care. Infant Mental Health Journal, 20, 30-41.

American Psychiatric Association (2013). Diagnostic and statistical manual of mental disorders: DSM-5. Arlington, VA: American Psychiatric Publishing.

Brooker, R.J., Buss, K.A., Lemery-Chalfant, K., Aksan, N., Davidson, R.J., \& Goldsmith, H.H. (2013). The development of stranger fear in infancy and toddlerhood: Normative development, individual differences, antecedents, and outcomes. Developmental Science, 16, 864-878.

Bruce, J., Tarullo, A.R., \& Gunnar, M.R. (2009). Disinhibited social behavior among internationally adopted children. Development and Psychopathology, 21, 157-171.

Carlson, E.A., Hostinar, C.E., Mliner, S.B., \& Gunnar, M.R. (2014). The emergence of attachment following early social deprivation. Development and Psychopathology, 26, 479-489.

Chisholm, K. (1998). A three-year follow-up of attachment and indiscriminate friendliness in children adopted from Romanian orphanages. Child Development, 69, 1092-1106.

Garvin, M.C., Tarullo, A.R., Van Ryzin, M., \& Gunnar, M.R. (2012). Post-adoption parenting and socioemotional development in post-institutionalized children. Development and Psychopathology, 24, 35-48.

Gershon, J. (2002). A meta-analytic review of gender differences in ADHD. Journal of Attention Disorders, 5, 143-154.

Gleason, M.M., Fox, N.A., Drury, S., Smyke, A., Egger, H.L., Nelson, C.A., ... \& Zeanah, C.H. (2011). Validity of evidencederived criteria for reactive attachment disorder: Indiscriminately social/disinhibited and emotionally withdrawn/inhibited types. Journal of the American Academy of Child and Adolescent Psychiatry, 50, 216-231.

Gleason, M.M., Fox, N.A., Drury, S.S., Smyke, A.T., Nelson, C.A., $\&$ Zeanah, C.H. (2014). Indiscriminate behaviors in previously institutionalized young children. Pediatrics, 133, e657-e665.

Hodges, J., \& Tizard, B. (1989). Social and family relationships of ex-institutional adolescents. Journal of Child Psychology and Psychiatry, 30, 77-97.

Julian, M.M. (2013). Age at adoption from institutional care as a window into the lasting effects of early experiences. Clinical Child and Family Psychology Review, 16, 101-145.

Kreppner, J., Kumsta, R., Rutter, M., Beckett, C., Castle, J., Stevens, S., \& Sonuga-Barke, E.J. (2010). IV. Developmental course of deprivation-specific psychological patterns: Early manifestations, persistence to age 15, and clinical features. Monographs of the Society for Research in Child Development, 75, 79-101.

Lawler, J.M., Hostinar, C.E., Mliner, S.B., \& Gunnar, M.R. (2014). Disinhibited social engagement in post-institutionalized children: Differentiating normal from atypical behavior. Development and Psychopathology, 26, 451-464.

Lyons-Ruth, K., Bureau, J.F., Riley, C.D., \& Atlas-Corbett, A.F (2009). Socially indiscriminate attachment behavior in the Strange Situation: Convergent and discriminant validity in relation to caregiving risk, later behavior problems, and attachment insecurity. Development and Psychopathology, 21, 355-372.

McArdle, J.J., \& Hamagami, F. (2001). Latent difference score structural models for linear dynamic analysis with incomplete longitudinal data. In L. Collins \& A. Sayer (Eds.), New methods for the analysis of change (pp. 137-175). Washington, DC: American Psychological Association.

O'Connor, T.G., Bredenkamp, D., Rutter, M., \& The ERA Study Team (1999). Attachment disturbances and disorders in children exposed to early severe deprivation. Infant Mental Health Journal, 20, 10-29.

O'Connor, T.G., \& Rutter, M. (2000). Attachment disorder behavior following early severe deprivation: Extension and longitudinal follow-up. Journal of the American Academy of Child and Adolescent Psychiatry, 39, 703-712.
Oliveira, P.S., Soares, I., Martins, C., Silva, J.R., Marques, S., Baptista, J., \& Lyons-Ruth, K. (2012). Indiscriminate behavior observed in the strange situation among institutionalized toddlers: Relations to caregiver report and to early family risk. Infant Mental Health Journal, 33, 187-196.

Onis, M.D., Onyango, A.W., Borghi, E., Siyam, A., Nishida, C., \& Siekmann, J. (2007). Development of a WHO growth reference for school-aged children and adolescents. Bulletin of the World Health Organization, 85, 660-667.

Pears, K.C., Fisher, P.A., Bruce, J., Kim, H.K., \& Yoerger, K. (2010). Early elementary school adjustment of maltreated children in foster care: The roles of inhibitory control and caregiver involvement. Child Development, 81, 1550-1564.

Roy, P., Rutter, M., \& Pickles, A. (2004). Institutional care: Associations between overactivity and lack of selectivity in social relationships. Journal of Child Psychology and Psychiatry, 45, 866-873.

Rutter, M., Colvert, E., Kreppner, J., Beckett, C., Castle, J., Groothues, C., ... \& Sonuga-Barke, E.J. (2007). Early adolescent outcomes for institutionally-deprived and nondeprived adoptees. I: Disinhibited attachment. Journal of Child Psychology and Psychiatry, 48, 17-30.

Rutter, M., Sonuga-Barke, E.J., Beckett, C., Castle, J., Kreppner, J., Kumsta, R., . . \& Gunnar, M.R. (2010). Deprivationspecific psychological patterns: Effects of institutional deprivation. Monographs of the Society for Research in Child Development, 75, 1-252.

Smyke, A.T., Dumitrescu, A., \& Zeanah, C.H. (2002). Attachment disturbances in young children. I: The continuum of caretaking casualty. Journal of the American Academy of Child and Adolescent Psychiatry, 41, 972-982.

Smyke, A.T., Koga, S.F., Johnson, D.E., Fox, N.A., Marshall, P.J., Nelson, C.A., \& Zeanah, C.H. (2007). The caregiving context in institution-reared and family-reared infants and toddlers in Romania. Journal of Child Psychology and Psychiatry, 48, 210218.

Smyke, A.T., Zeanah, C.H., Fox, N.A., Nelson, C.A., \& Guthrie, D. (2010). Placement in foster care enhances quality of attachment among young institutionalized children. Child Development, 81, 212-223.

Smyke, A.T., Zeanah, C.H., Gleason, M.M., Drury, S.S., Fox, N.A., Nelson, C.A., \& Guthrie, D. (2012). A randomized controlled trial comparing foster care and institutional care for children with signs of reactive attachment disorder. American Journal of Psychiatry, 169, 508-514.

Sroufe, L.A. (1977). Wariness of strangers and the study of infant development. Child Development, 48, 731-746.

Tapp, J. (2003). Procoder for digital video [Computer software]. Nashville, TN: Vanderbilt Kennedy Center.

Tizard, B. (1977). Adoption: A second chance. London: Open Books.

Tizard, B., \& Rees, J. (1975). The effect of early institutional rearing on the behaviour problems and affectional relationships of four-year-old children. Journal of Child Psychology and Psychiatry, 16, 61-73.

Zeanah, C.H., \& Gleason, M.M. (2015). Annual research review: Attachment disorders in early childhood-clinical presentation, causes, correlates, and treatment. Journal of Child Psychology and Psychiatry, 56, 207-222.

Zeanah, C.H., Scheeringa, M., Boris, N.W., Heller, S.S., Smyke, A.T., \& Trapani, J. (2004). Reactive attachment disorder in maltreated toddlers. Child Abuse and Neglect, 28, 877-888.

Zeanah, C.H., Smyke, A.T., \& Dumitrescu, A. (2002). Attachment disturbances in young children. II: Indiscriminate behavior and institutional care. Journal of the American Academy of Child and Adolescent Psychiatry, 41, 983-989.

Zeanah, C.H., Smyke, A.T., Koga, S.F., \& Carlson, E. (2005). Attachment in institutionalized and community children in Romania. Child Development, 76, 1015-1028.

Accepted for publication: 11 May 2016

First published online: 27 July 2016 